\title{
Posaconazole achieves prompt recovery of voriconazole-induced liver injury in a case of invasive aspergillosis
}

This article was published in the following Dove Press journal: Infection and Drug Resistance

\author{
Javier Martínez-Casanova' \\ Nuria Carballo' \\ Sonia Luque' \\ Luisa Sorli \\ Santiago Grau'
}

'Pharmacy Department, Hospital del Mar, Barcelona, Spain; ${ }^{2}$ Infectious Diseases Department, Hospital del Mar, Barcelona, Spain
Correspondence: Santiago Grau Servicio de Farmacia, Hospital del Mar, Passeig Marítim 25-29, 08025 Barcelona, Spain

Tel +3493248 3152

Email SGrau@parcdesalutmar.cat

\begin{abstract}
Azole antifungals have frequently been linked to the presence of hepatotoxicity, but there is scarce information on cross-toxicity between these drugs or on the possibility of using some of them when this type of toxicity occurs. We report the case of a 64-year-old man with invasive aspergillosis (IA) leading to spondylodiscitis with neurological involvement. Early management included intravenous (iv) voriconazole, which had to be interrupted after 1 week due to liver damage. Therapeutic drug monitoring (TDM) of voriconazole showed that the plasma concentration was within the therapeutic range. However, it was replaced by a combination therapy of oral posaconazole plus iv caspofungin. Posaconazole allowed normalization of liver enzymes. After finishing posaconazole monotherapy on an outpatient basis, the patient made a full recovery. This case report provides further evidence that oral posaconazole is safe and effective as rescue therapy after the appearance of voriconazole-induced liver toxicity.
\end{abstract}

Keywords: case report, antifungal agents, hepatotoxicity, therapeutic drug monitoring

\section{Introduction}

Antimicrobial-related toxicity is an important issue in clinical practice, especially in cases involving multidrug-resistant bacteria or difficult-to-treat microorganisms, including fungi. Despite the broad evidence of azole-related hepatotoxicity, ${ }^{1}$ there is limited information on the potential cross-reactivity between these agents, creating uncertainty when establishing a therapeutic approach. Current guidelines recommend the use of triazoles for a minimum of 6-12 weeks for the treatment of invasive aspergillosis (IA). Among triazoles, voriconazole is the recommended primary treatment. If there is refractory or progressive aspergillosis, posaconazole is recommended as salvage therapy, with a switch to an alternative class of antifungal being advised if the patient exhibits an adverse event attributable to the previous agent. ${ }^{2}$ The latter point is controversial, since a recent review of antifungal toxicity suggested that there was growing evidence for the possibility of replacing voriconazole with posaconazole in cases of hepatic toxicity. ${ }^{3}$ However, as shown in the following, the information is scarce and shows substantial limitations. In this scenario, we described the case of a patient who received posaconazole as rescue therapy after voriconazole-induced hepatotoxicity. We also reviewed the existing literature on this topic to emphasize new aspects regarding liver toxicity and the safety of switching these antifungals.

\section{Case report}

A 64-year-old man with persistent low back pain associated with disabling muscular weakness was attended in the emergency department. Magnetic resonance imaging of 
his back and head revealed spinal and intracerebral damage, initially suggesting infectious spondylodiscitis or a neoplastic process. Since the patient was immunocompetent and microbiological cultures from several fine-needle aspiration biopsies were repeatedly negative, an infectious etiology was initially excluded. The low incidence of IA in this population group was an additional challenge in terms of diagnosis and decision making on treatment. However, histopathological samples did not show tumor cells, and positron-emission tomography suggested an infectious origin of the lesions.

Six weeks after admission, the patient's clinical status worsened with the onset of neurological symptoms. An open vertebral biopsy revealed isolation of Aspergillus terreus, and the same etiology was assumed for the brain lesions. Antifungal treatment was started with intravenous (iv) voriconazole ( $6 \mathrm{mg} / \mathrm{kg}$ twice daily as a loading dose followed by $4 \mathrm{mg} / \mathrm{kg}$ twice daily as a maintenance dose). At that time, liver function tests were as follows: gamma-glutamyl transpeptidase (GGT) $72 \mathrm{UI} / \mathrm{L}$, alkaline phosphatase (ALP) $122 \mathrm{UI} / \mathrm{L}$, alanine transaminase (ALT) $26 \mathrm{UI} / \mathrm{L}$, aspartate transaminase (AST) $18 \mathrm{UI} / \mathrm{L}$ and total bilirubin $0.2 \mathrm{mg} / \mathrm{dL}$ (Figure 1).

After 7 days of treatment with voriconazole, the ALP and GGT values increased to $307 \mathrm{UI} / \mathrm{L}$ and $631 \mathrm{UI} / \mathrm{L}$, respectively, while ALT and AST levels were only mildly elevated (65 UI/L and $63 \mathrm{UI} / \mathrm{L}$, respectively). The patient's bilirubin level remained within the normal range throughout the treatment $(0.3 \mathrm{mg} / \mathrm{dL})$. Concomitant medication was reviewed, and none of the administered drugs, except voriconazole, appeared to be potentially hepatotoxic. In addition, the Naranjo algorithm ${ }^{4}$ was applied to better confirm the potential causal relationship between voriconazole and this pattern of dissociated cholestasis and hypertransaminasemia. This is a validated algorithm used to perform a causation analysis between administration of a drug and the occurrence of an adverse effect. Its result (seven out of 13) revealed a probable imputability and allowed the exclusion of the concomitant medication. Additionally, drug-drug interactions were also checked; there were no concurrent medications that could affect hepatic metabolism of voriconazole via the cytochrome P450 enzymatic complex and lead to serum-level elevation. ${ }^{5}$ Therapeutic drug monitoring (TDM) of the trough concentration was performed under steady-state conditions using a validated high-performance liquid chromatography (HPLC) method. The plasma concentration was $4.12 \mathrm{mg} / \mathrm{L}$, a value within the reported therapeutic range $(1-5.5 \mathrm{mg} / \mathrm{L}),{ }^{6}$ excluding the possibility of a concentration-toxicity relationship and mandating immediate drug discontinuation with no possibility of readministration. As recommended for difficult-to-treat infections, ${ }^{7}$ voriconazole was replaced by combination antifungal therapy, consisting of oral posaconazole suspension

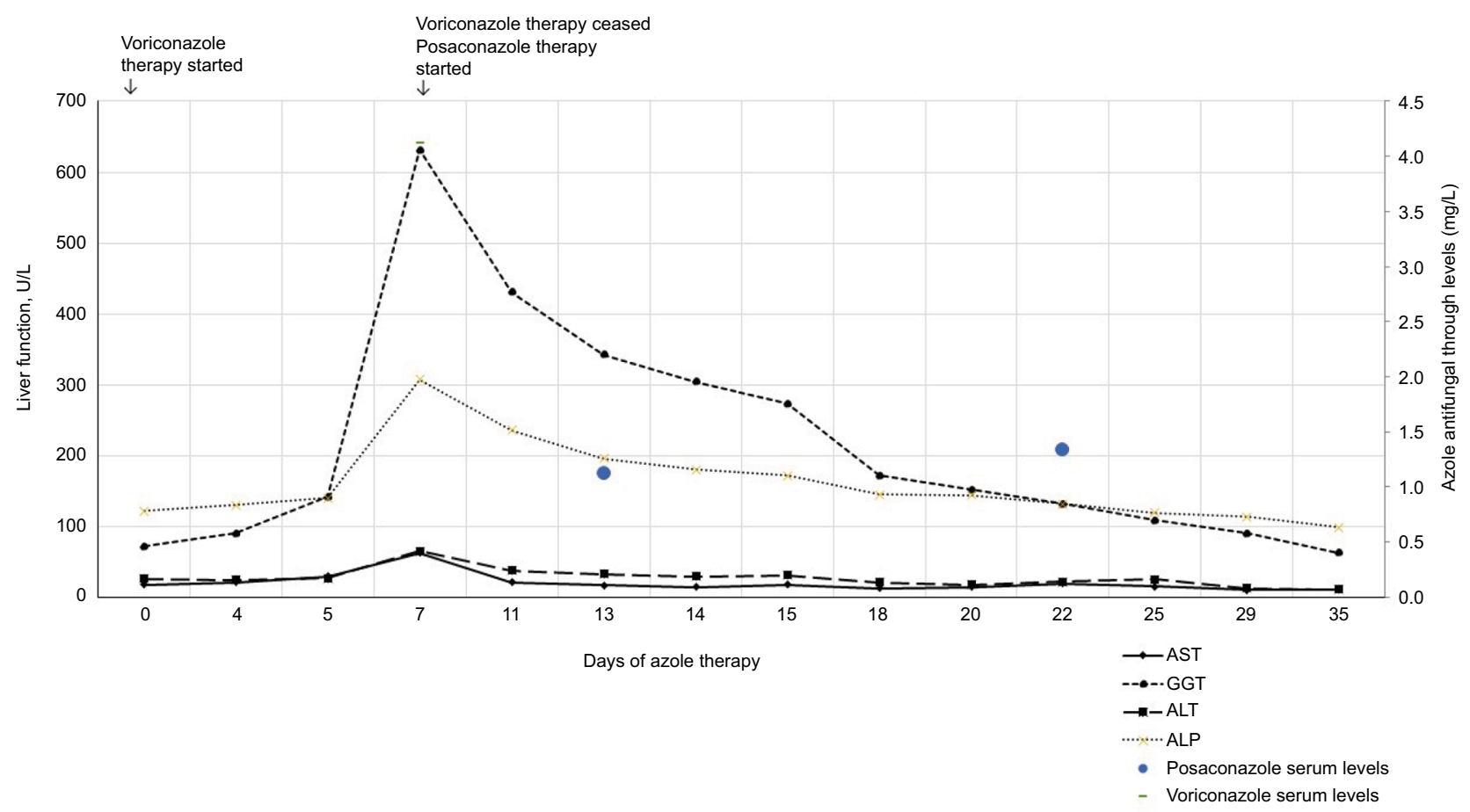

Figure I Changes in liver function test values and azole antifungal serum levels during antifungal therapy.

Notes: Liver enzyme normal values (UI/L): GGT 8-16, ALP 40-129, ALT 7-4I, AST 10-40. Serum bilirubin values are not represented, always kept within the normal range (0.2-I.2 mg/dL).

Abbreviations: GGT, gamma-glutamyl transpeptidase; ALP, alkaline phosphatase; ALT, alanine transaminase; AST, aspartate transaminase. 
(200 mg/6 h) plus iv caspofungin $(50 \mathrm{mg} / 24 \mathrm{~h}$ after a loading dose of $70 \mathrm{mg}$ ). Despite demonstration of the better bioavailability of delayed-release posaconazole tablets, posaconazole suspension was prescribed because the posaconazole tablet formulation was not included in our hospital formulary.

After 6 days of treatment of combined antifungal therapy, ALP and GGT levels decreased to $196 \mathrm{UI} / \mathrm{L}$ and $342 \mathrm{UI} / \mathrm{L}$, respectively, and a similar trend was observed for ALT and AST values, which decreased to $33 \mathrm{UI} / \mathrm{L}$ and $17 \mathrm{UI} / \mathrm{L}$, respectively. At that moment, TDM of both antifungals was performed and posaconazole and caspofungin plasma levels were $1.12 \mathrm{mg} / \mathrm{L}$ and $9.7 \mathrm{mg} / \mathrm{L}$, respectively, which were both within the therapeutic range for the treatment of invasive fungal infections (1-3.75 mg/L $\mathrm{L}^{6,8}$ and $1.4-12.1 \mathrm{mg} / \mathrm{L},{ }^{9}$ respectively). Given that the suspension formulation of posaconazole has variable bioavailability and that steady-state levels may not be reached until 7-10 days of treatment, ${ }^{10}$ a second plasma sample was obtained after 15 days of treatment initiation and the concentration was $1.34 \mathrm{mg} / \mathrm{L}$, which confirmed the previous result.

During treatment with posaconazole plus caspofungin, a steady improvement was observed in the patient's liver function parameters and acute-phase reactants. After 17 days of voriconazole discontinuation, the hepatotoxicity was resolved (ALP, GGT, ALT and AST levels fell to $119 \mathrm{UI} / \mathrm{L}, 108 \mathrm{UI} / \mathrm{L}, 25 \mathrm{UI} / \mathrm{L}$ and $15 \mathrm{UI} / \mathrm{L}$, respectively). After 1 month of treatment with this combination, the patient's clinical status improved and caspofungin was stopped. The patient was finally discharged with oral posaconazole $200 \mathrm{mg} / 6 \mathrm{~h}$ monotherapy, which was completed on an outpatient basis for 2 more weeks until completion of 12 weeks of antifungal treatment. At the next medical visit, liver enzymes continued to be within normal values, a magnetic resonance scan showed clear improvement in the brain abscess and the patient was in good health, able to stand up and walk.

Written informed consent was obtained from the patient to have the case details published.

\section{Discussion}

Here, we describe the case of a patient with IA initially treated with voriconazole, who, after the onset of hepatotoxicity, was treated with posaconazole as rescue treatment and showed good outcome and reversal of liver damage.

Invasive fungal infections are associated with high morbidity and mortality. Therefore, early and adequate concentration exposure to the appropriate antifungal agent is crucial to achieve a clinical cure and eradicate the pathogen. Voriconazole and posaconazole are triazoles with broad spectrum antifungal activity and are recommended as first-line and salvage therapy for IA. Both are metabolized in the liver, but voriconazole involves the CYP complex and posaconazole is metabolized by glucuronidation. Both antifungals are potent inhibitors of CYP3A4 (and voriconazole also inhibits CYP2C19), which may result in many potential drug-drug interactions. ${ }^{2}$ Owing to its highly variable pharmacokinetics, TDM for both antifungals is widely recommended to enhance therapeutic efficacy and to avoid potential associated toxicity. ${ }^{2}$ Moreover, posaconazole suspension shows saturable absorption with reported subtherapeutic levels, which strengthens the recommendation for TDM. ${ }^{11}$

A cholestatic pattern of liver toxicity, defined as a rise of ALP more than twice the upper limit of normal, was observed in our patient. ${ }^{1}$ The reported frequency of liver toxicity induced by antifungal therapy varies widely and is highest with azole antifungals. For these drugs, the severity of liver toxicity ranges from mild abnormalities in liver function tests with no clinical implications to severe liver injury including fulminant hepatic failure. ${ }^{3}$ The pattern is usually hepatocellular, which is not in our case, but can also be cholestatic, or both. ${ }^{3}$ A meta-analysis evaluating the hepatotoxicity of antifungals concluded that elevation of serum liver tests was more frequent for voriconazole than for any other azole and occurred in $19.7 \%$ of patients, although it did not always lead to treatment withdrawal. ${ }^{12}$ Another study reported that $6.3 \%$ of patients treated with voriconazole had to interrupt treatment due to hepatotoxicity. ${ }^{13}$ In addition, this adverse effect does not follow a specific pattern and usually appears during the first month of treatment. ${ }^{14}$ Data from clinical trials indicate that posaconazole is well tolerated, with the most common adverse effects being headache, dry mouth and dizziness. ${ }^{15}$ Nevertheless, liver alterations, including severe cholestasis and liver failure, have been described. ${ }^{8}$ Recently, a review of hepatotoxicity induced by antifungal agents attributed an incidence rate of $12.7 \%$ of increased transaminases to posaconazole. ${ }^{1}$ There are no specific recommendations on the approach to be adopted regarding the possibility of substituting one azole for another when liver toxicity occurs.

To date, the largest series on the use of posaconazole as rescue therapy after previous azole therapy is a retrospective study published by Heinz et al, ${ }^{16}$ which included 40 patients treated with posaconazole after a previous antifungal therapy with voriconazole. The main cause of treatment discontinuation in $23(57.5 \%)$ patients was the lack of response to voriconazole, while an increase in transaminases was the cause of treatment discontinuation in only four (10.0\%) patients. A limitation of that study was that the authors may have erroneously attributed the hepatotoxicity to voriconazole, while it could have been caused by other concomitant drugs. 
Dilokpattanamongkol et al ${ }^{17}$ also recently published the case of a patient with IA caused by $A$. terreus successfully treated with posaconazole after treatment failure with voriconazole. Unlike our patient, this patient showed alterations in liver function parameters prior to the initiation of voriconazole treatment. Furthermore, the voriconazole treatment was discontinued due to the risk of hepatotoxicity related to the vehicle containing the iv formulation, sulfobutylether$\beta$-cyclodextrin, and not to the voriconazole itself.

Foo and Gottlieb ${ }^{18}$ also previously reported a case of lack of cross-hepatotoxicity between voriconazole and posaconazole in the treatment of a patient with intracerebral aspergillosis. Similar to our case, liver function tests were reported with accuracy, showing a clear resolution and recovery, and a possible correlation between voriconazole blood levels $(0.6 \mathrm{mg} / \mathrm{L})$ and hepatotoxicity was ruled out. Unlike our case, both the onset and normalization of liver function tests after voriconazole initiation and discontinuation were slow (43 days and 4 weeks, respectively) compared with the presentation and resolution of this adverse effect in our patient ( 7 days and 17 days, respectively). In terms of clinical efficacy, both cases support the use of posaconazole in central nervous system infections.

No clear explanation has yet been reported on the mechanism of toxicity of azolic agents. Nevertheless, a recent in vitro study proposed that voriconazole and posaconazole have different mechanisms of toxicity, ${ }^{19}$ which would support the observed lack of cross-toxicity and the use of one or the other drug in cases of previous treatment failure.

\section{Conclusion}

The available literature on cross-toxicity between voriconazole and posaconazole is scarce. Most of the information on this topic seems questionable and shows substantial limitations. Through the implementation of the Naranjo algorithm ${ }^{4}$ and the availability of plasma concentrations of the three antifungal agents used, this case report overcomes these limitations and strengthens the results. Thus, we stress the safety and effectiveness of posaconazole in patients with difficult-to-treat infections and voriconazole-induced hepatotoxicity. Future studies should aim to identify the risk factors influencing the occurrence of early versus delayed liver toxicity and recovery.

\section{Acknowledgment}

Medical writing assistance was provided by Gail Craigie and was funded by Merck Sharp \& Dohme Spain, with no influence on the reporting of this clinical case.

\section{Disclosure}

The authors report no conflicts of interest in this work.

\section{References}

1. Kyriakidis I, Tragiannidis A, Munchen S, Groll AH. Clinical hepatotoxicity associated with antifungal agents. Expert Opin Drug Saf. 2016;16(2):1-17.

2. Patterson TF, Thompson GR, Denning DW, et al. Practice guidelines for the diagnosis and management of aspergillosis: 2016 update by the infectious diseases society of America. Clin Infect Dis. 2016;63(4):e1-e60.

3. Tverdek FP, Kofteridis D, Kontoyiannis DP. Antifungal agents and liver toxicity: a complex interaction. Expert Rev Anti Infect Ther. 2016;14(8):765-776.

4. Naranjo CA, Busto U, Sellers EM, et al. A method for estimating the probability of adverse drug reactions. Clin Pharmacol Ther. 1981;30(2):239-245.

5. Baxter K, editors. Stockley's Drug Interactions. 8th ed. London: Pharmaceutical Press; 2008.

6. Ashbee HR, Barnes RA, Johnson EM, Richardson MD, Gorton R, Hope WW. Therapeutic drug monitoring (TDM) of antifungal agents: guidelines from the British Society for medical mycology. JAntimicrob Chemother. 2014;69(5):1162-1176.

7. Hatipoglu N, Hatipoglu H. Combination antifungal therapy for invasive fungal infections in children and adults. Expert Rev Anti Infect Ther. 2013;11(5):523-535.

8. EMA. Posaconazole (Noxafil) - Assessment Report. Vol. 44. London: 2013.

9. Muilwijk EW, Lempers VJC, Burger DM, et al. Impact of special patient populations on the pharmacokinetics of echinocandins. Expert Rev Anti Infect Ther. 2015;13(6):799-815.

10. Dekkers BGJ, Bakker M, van der Elst KCM, et al. Therapeutic Drug Monitoring of Posaconazole: an Update. Curr Fungal Infect Rep. 2016;10(2):51-61.

11. van der Elst KCM, Brouwers CHS, van den Heuvel ER, et al. Subtherapeutic posaconazole exposure and treatment outcome in patients with invasive fungal disease. Ther Drug Monit. 2015;37(6):766-771.

12. Wang JL, Chang CH, Young-Xu Y, Chan KA. Systematic review and meta-analysis of the tolerability and hepatotoxicity of antifungals in empirical and definitive therapy for invasive fungal infection. Antimicrob Agents Chemother. 2010;54(6):2409-2419.

13. Zonios D, Yamazaki H, Murayama N, et al. Voriconazole metabolism, toxicity, and the effect of cytochrome P450 2C19 genotype. J Infect Dis. 2014;209(12):1941-1948.

14. Denning DW, Ribaud P, Milpied N, et al. Efficacy and safety of voriconazole in the treatment of acute invasive aspergillosis. Clin Infect Dis. 2002;34(5):563-571.

15. Moton A, Krishna G, Wang Z. Tolerability and safety profile of posaconazole: evaluation of 18 controlled studies in healthy volunteers. J Clin Pharm Ther. 2009;34(3):301-311.

16. Heinz WJ, Egerer G, Lellek H, Boehme A, Greiner J. Posaconazole after previous antifungal therapy with voriconazole for therapy of invasive aspergillus disease, a retrospective analysis. Mycoses. 2013;56(3):304-310.

17. Dilokpattanamongkol P, Panusitthikorn P, Boonprasert R, Chayakulkeeree M, Rotjanapan P. A case report of intravenous posaconazole in hepatic and renal impairment patient with invasive Aspergillus terreus infection: safety and role of therapeutic drug monitoring. BMC Pharmacol Toxicol. 2017;18(1):8.

18. Foo H, Gottlieb T. Lack of cross-hepatotoxicity between voriconazole and posaconazole. Clin Infect Dis. 2007;45(6):803-805.

19. Haegler P, Joerin L, Krähenbühl S, Bouitbir J. Hepatocellular toxicity of imidazole and triazole antimycotic agents. Toxicol Sci. 2017;157(1):183-195. 
Infection and Drug Resistance is an international, peer-reviewed openaccess journal that focuses on the optimal treatment of infection (bacterial, fungal and viral) and the development and institution of preventive strategies to minimize the development and spread of resistance. The journal is specifically concerned with the epidemiology of antibiotic resistance and the mechanisms of resistance development and diffusion in both hospitals and the community. The manuscript management system is completely online and includes a very quick and fair peerreview system, which is all easy to use. Visit http://www.dovepress.com/ testimonials.php to read real quotes from published authors.

Submit your manuscript here: https://www.dovepress.com/infection-and-drug-resistance-journal 